\title{
Invited Article Foreword
}

The Invited Article section of this issue consists of one article. "Bounds on Monotone Switching Networks for Directed Connectivity" by Aaron Potechin won a Machtey Award for best student paper at the 51st Annual IEEE Symposium on Foundations of Computer Science (FOCS'10) in 2010. We want to thank the FOCS'10 Program Committee for their help in selecting this invited article, as well as the editor Luca Trevisan, who handled it.

Eva Tardos

Editor-in-Chief 